\title{
Thermodynamic properties of the XX model in a chain with a period two and three coupling
}

\author{
Julio Abad \\ Departamento de Física Teórica, Facultad de Ciencias, \\ Universidad de Zaragoza, 50009 Zaragoza, Spain.
}

(Dated: 31st December 2018)

\begin{abstract}
The exact solutions for the energy spectrum of the XX model with a periodic coupling and an external transverse magnetic field $h$ are obtained. The diagonalization procedure is discussed, and analytical and numerical solutions are given. Using the solutions for period-two coupling, the free energy, entropy, and specific heat are calculated as functions of temperature and applied transverse external magnetic field. Their expressions show that below a particular value $v$ and above a value $u$ of the magnetic field $|h|$, the entropy and the specific heat vanish exponentially in the low temperature limit.
\end{abstract}

PACS numbers: 05.70.Fh, 05.70.Jk, 75.10Pq 


\section{INTRODUCTION}

The physics of one dimensional chains provide a good example for the rigourous study of some properties of quantum magnetic systems. They are known to be useful for analysing many-body problems. Recently, real, model quasi-1D systems of atoms have become the object of experiments. From a experimental point of view, there are many quasi one dimensional compounds (such as the organic series $(\mathrm{BCPTTF})_{2} X$ with $\left(X=\mathrm{AsF}_{6}\right.$, $\mathrm{PF}_{6}$ ), the cuprate $\mathrm{CuGeO}_{3}$, or the $(\mathrm{VO})_{2} \mathrm{P}_{2} \mathrm{O}_{7}$ compounds, as well as other series as the $\mathrm{TTFMS}_{4} \mathrm{C}_{4}\left(\mathrm{CF}_{3}\right)_{4}$ with $M=\mathrm{Cu}, \mathrm{Au}, \mathrm{Pt}$, or $\left.\mathrm{Ni}[1-7]\right)$. These are properly described as Peierls spin-chains.

The experimental discovery of quasi-crystals $[8,9]$ has stimulated intense activity in the study of the periodic and aperiodic chains, with the aim of understanding their physical properties [10].

Recently, new, unusual properties in low-dimensional magnetic materials have been found [11-13] that can be explained in terms of many-body behaviour. It seems to be responsible for the occurrence of magnetization plateaus as a function of external magnetic fields $[14,15]$.

The procedure for the study of this type of structures was initiated by Leib et al. [16], by using the well known Jordan-Wigner transformation [17], and assuming that the chain is finite with set boundary conditions.

One of the most important systems is the 1D $X Y$ model $(S=1 / 2)$, introduced by Leib et al. [16]. It plays an important role in the description of many-body problems since it can be solved exactly.

Two early examples of studies of ground state properties (e.g. susceptibility) for the $X Y$ model with alternating interaction are those by Perk et al. [18], and Taylor and Müller [19].

Quantum critical phenomena in random $X Y$ chains have been studied using renormalization group methods [20], and by numerical methods [21, 22].

Recently, Derzhko et al. [23-25] have described the thermodynamical behaviour and properties of the ground state of periodic non-uniform $X Y$ chains in a transverse field. Also, thermodynamic properties in one dimensional superlattices have been treated by de Lima et al. [26-28]. An excellent review is provided in Ref. [29].

As mentioned previously, systems of this type are interesting due to being strongly af- 
fected by quantum fluctuations, and by their apparent simplicity. For example, low dimensional electronic materials are known to be very sensitive to structural distortions that are driven by electron-phonon interactions. These break the symmetry of the original ground state, which results in a new lower energy state where the electrons and ions are shifted from their original positions in a regular manner. This creates a periodic variation of the charge density that is called a charge density wave. This is the well know Peierls instability which opens a gap at the Fermi surface of the 1D electronic chain, transforming a metal into a semiconductor. A similar effect is expected for 1D spin chains, which are unstable against lattice vibrations.

The problem considered here is a homogeneous chain where the structural distortions of the lattice are taken into account through a periodic coupling between a given site and its neighbouring sites. An external transverse magnetic field $h$ is also applied. This permits us to study the diverse phases of the system. Thus, the Hamiltonian is,

$$
H=-\sum_{i=1}^{N} J_{i, i+1}\left(S_{i}^{x} S_{i+1}^{x}+S_{i}^{y} S_{i+1}^{y}\right)-h \sum_{i=1}^{N} S_{i}^{z}
$$

where

$$
J_{i, i+1}=J_{i+a, i+a+1}=u+v \cos \left(\frac{2 \pi m}{N} i\right),
$$

and the quantum spin operators are $S_{i}^{c}=(1 / 2) \sigma_{i}^{c}$ (where $\sigma_{i}^{c}$ are the Pauli matrices at the site $i$ ), and $a=N / m$ is an integer.

This work is divided in four sections. In section II we begin with the Hamiltonian (1). The Jordan-Wigner transformation is applied, then the Hamiltonian is diagonalized with a superposition between creation operators. The same superposition is used between the corresponding destruction operators. This superposition is possible due to the Hamiltonian being isotropic, thus preserving the total transverse spin $S_{z}=\sum_{i} S_{i}^{z}$.

In section III, analytical results for the energy bands and gaps are given for the integer values $a=2$ and 3. Graphs are provided that show numerical results for higher values of $a$. The degeneration of the bands into single levels is discussed.

In section IV, thermodynamic quantities - free energy, entropy and specific heat-and their behaviour are discussed. The free energy per site is calculated as a function of temperature. The other thermodynamic properties are subsequently derived from this. Graphs showing the free energy and entropy are given. 
An interesting result is found by taking the low temperature limit. It is shown that the entropy and the specific heat possess local maxima at $|h|=v$ and $|h|=u$. They vanish exponentially with temperature for $|h|<v$ and $|h|>u$. Both functions at the maxima vary as a square root of temperature, while in between they are proportional to temperature.

\section{THE MODEL}

The simplest solvable model (see for example Ref. [30]) is the isotropic spin $1 / 2 X Y$ model. This model is based on a one dimensional lattice with a uniform interaction strength $J$ between nearest neighbour sites, and uses periodic boundary conditions. As explained in the introduction, a correction to this Hamiltonian is provided by introducing a periodic strength, which simulates a vibration at the sites. Hence, the Hamiltonian from $(1,2)$ is

$$
H=-\sum_{i=1}^{N}\left[u+v \cos \left(\frac{2 \pi m}{N} i\right)\right]\left(S_{i}^{x} S_{i+1}^{x}+S_{i}^{y} S_{i+1}^{y}\right)-h S_{i}^{z}
$$

where $u$ is a uniform coupling and $v$ is the amplitude of the periodic coupling.

The spin operators $S_{i}$ act on a two dimensional space $\eta_{i}=C^{2}$ where we take the eigenstates of $S^{3}$ as a base. The total third component of the spin,

$$
S_{z}=\sum_{i=1}^{N} S_{i}^{z}
$$

is a conserved quantity that can be used to describe the states of the system.

The first task is to transform the Hamiltonian (3) into a fermionic Hamiltonian by means of the Jordan-Wigner transformation [17]. This is achieved by first defining the fermionic operators:

$$
\begin{aligned}
a_{l} & =K(l) S_{l}^{-}, \\
a_{l}^{\dagger} & =K(l) S_{l}^{+},
\end{aligned}
$$

where $S_{l}^{ \pm}=\left(S_{l}^{x} \pm i S_{l}^{y}\right)$, and

$$
K(l)=\exp \left(i \pi \sum_{j=1}^{l-1} S_{j}^{+} S_{j}^{-}\right)=\prod_{j=1}^{l-1}\left(-2 S_{j}^{z}\right) .
$$

The new Hamiltonian and the conserved spin component $S_{z}$ become,

$$
H=-\sum_{n=1}^{N}\left\{\left[u+v \cos \left(\frac{2 \pi}{a} n\right)\right] \frac{1}{2}\left(a_{n}^{\dagger} a_{n+1}+a_{n+1}^{\dagger} a_{n}\right)-h\left(a_{n}^{\dagger} a_{n}-\frac{1}{2} I\right)\right\}
$$




$$
S_{z}=\sum_{n=1}^{N}\left(a_{n}^{\dagger} a_{n}-\frac{1}{2} I\right)=N_{f}-\frac{N}{2} I
$$

$I$ is the unity operator, and $N_{f}=\sum_{n=1}^{N}\left(a_{n}^{\dagger} a_{n}\right)$ is the total fermionic number, which is also conserved.

These $a$ and $a^{\dagger}$ operators are true fermionic operators in the sense that $\left\{a_{l}^{\dagger}, a_{m}\right\}=\delta_{l, m}$ and $\left\{a_{l}^{\dagger}, a_{m}^{\dagger}\right\}=0=\left\{a_{l}, a_{m}\right\}$. Furthermore, since

$$
\sigma_{l}^{z}=2 a_{l}^{\dagger} a_{l}-1
$$

the periodic boundary conditions require that

$$
\begin{array}{lll}
a_{N+1}=-a_{1}, & a_{N+1}^{\dagger}=-a_{1}^{\dagger} & \text { for states on which } N_{f} \text { is even; } \\
a_{N+1}=a_{1}, & a_{N+1}^{\dagger}=a_{1}^{\dagger} & \text { for states on which } N_{f} \text { is odd. }
\end{array}
$$

The next step is to transform the system to momentum space by performing a Fourier transformation using new fermionic operators $b_{j}$ and $b_{j}^{\dagger}$. These are related to the $a_{j}$ and $a_{j}^{\dagger}$ by

$$
\begin{aligned}
& b_{j}=\frac{1}{\sqrt{N}} \sum_{l=1}^{N} \exp [i k(j) l] a_{l}, \\
& a_{l}=\frac{1}{\sqrt{N}} \sum_{j=1}^{N} \exp [-i k(j) l] b_{j},
\end{aligned}
$$

and hermitian conjugate, where

$$
k(j)=\frac{2 \pi}{N}\left(j-\frac{\varepsilon}{2}\right) \text { with }\left\{\begin{array}{l}
\varepsilon=0 \text { if } N_{f} \text { is odd, } \\
\varepsilon=1 \text { if } N_{f} \text { is even, } \quad j=1, \cdots, N .
\end{array}\right.
$$

The new operators $b_{j}$ and $b_{j}^{\dagger}$ destroy or create a fermion with momentum $k(j)$.

In terms of these operators, the Hamiltonian (8) is

$$
\begin{aligned}
H= & -u \sum_{j=1}^{N} \cos [k(j)] b_{j}^{\dagger} b_{j} \\
& -\frac{v}{2} \sum_{j=1}^{N} \cos \left[k\left(j+\frac{m}{2}\right)\right]\left(e^{i m \pi / N} b_{j}^{\dagger} b_{j+m}+e^{-i m \pi / N} b_{j+m}^{\dagger} b_{j}\right)-h\left(\frac{N}{2}-\sum_{j=1}^{N} b_{j}^{\dagger} b_{j}\right)(1
\end{aligned}
$$

A particular case is the alternating chain that occurs when $m=N / 2$. In this case, the Hamiltonian is

$$
\begin{aligned}
H= & -u \sum_{j=1}^{N} \cos [k(j)] b_{j}^{\dagger} b_{j} \\
& -i v \sum_{j=1}^{N} \sin [k(j)] b_{j}^{\dagger} b_{j+N / 2}-h\left(\frac{N}{2}-\sum_{j=1}^{N} b_{j}^{\dagger} b_{j}\right) .
\end{aligned}
$$


The commutation rule of the Hamiltonian with the $b_{j}^{\dagger}$ operator yields

$$
\begin{aligned}
{\left[H, b_{j}^{\dagger}\right]=} & \{h-u \cos [k(j)]\} b_{j}^{\dagger} \\
& -\frac{v}{2} \cos \left[k\left(j-\frac{m}{2}\right)\right] e^{i m \pi / N} b_{j-m}^{\dagger}-\frac{v}{2} \cos \left[k\left(j+\frac{m}{2}\right)\right] e^{-i m \pi / N} b_{j+m}^{\dagger},
\end{aligned}
$$

Therefore, $b_{j}$ gives the hermitian conjugate of this. As we can see, these commutation rules mix the operators $b_{j}$ and $b_{j+m}$. Hence, we can redefine the index of $b_{j}$ in a new form,

$$
b_{j}=b_{l, s} \text { with } j=l+s m \text { and } l=1, \cdots, m \text { and } s=0, \cdots, a-1 .
$$

The periodic conditions are $b_{l, a}=b_{l, 0}$, and $b_{l,-1}=b_{l, a-1}$.

The sums in the index $j$ will be transformed

$$
\sum_{j=1}^{N} \rightarrow \sum_{l=1}^{m} \sum_{s=0}^{a-1} \text { with } a=\frac{N}{m},
$$

and the functions $k(j)$

$$
k(j)=k^{1}(l, s)=k(l)+\frac{2 \pi s}{a}
$$

Hence, the commutation rule (16) takes the form

$$
\begin{aligned}
{\left[H, b_{l, s}^{\dagger}\right]=} & \left\{h-u \cos \left[k^{1}(l, s)\right]\right\} b_{l, s}^{\dagger} \\
& -\frac{v}{2} \cos \left[k^{1}(l, s)-\frac{\pi}{a}\right] e^{i \pi / a} b_{l, s-1}^{\dagger}-\frac{v}{2} \cos \left[k^{1}(l, s)+\frac{\pi}{a}\right] e^{-i \pi / a} b_{l, s+1}^{\dagger},
\end{aligned}
$$

that we will write as

$$
\left[H, b_{l, s}^{\dagger}\right]=\sum_{r=0}^{a-1} c_{r, s}^{l} b_{l, r}^{\dagger}
$$

with

$$
\begin{aligned}
c_{s, s}^{l} & =h-u \cos \left[k^{1}(l, s)\right], \\
c_{s-1, s}^{l} & =-\frac{v}{2} \cos \left[k^{1}(l, s)-\frac{\pi}{a}\right] e^{i \pi / a}, \\
c_{s+1, s}^{l} & =-\frac{v}{2} \cos \left[k^{1}(l, s)+\frac{\pi}{a}\right] e^{-i \pi / a}, \\
c_{a-1,0}^{l} & =-\frac{v}{2} \cos \left[k^{1}(l, 0)-\frac{\pi}{a}\right] e^{i \pi / a}, \\
c_{0, a-1}^{l} & =-\frac{v}{2} \cos \left[k^{1}(l, 0)-\frac{\pi}{a}\right] e^{-i \pi / a}, \text { and } \\
c_{r, s}^{l} & =0 \text { otherwise. }
\end{aligned}
$$

For each $l$, these equations represent the elements of a hermitian $a \times a$ matrix that we write as

$$
C^{l}=\left\{c_{r, s}^{l}\right\}
$$


The eigenvalues of the these matrices yield the energy spectrum of the system.

The case $m=N / 2$ does not follow from (20) and must be calculated directly from the commutation relation with $a=2$. The results for the elements of $C^{l}$ are

$$
\begin{aligned}
& c_{0,0}^{l}=h-u \cos [k(l)], \\
& c_{1,1}^{l}=h+u \cos [k(l)], \\
& c_{0,1}^{l}=-i v \sin [k(l)] .
\end{aligned}
$$

The Hamiltonian is diagonalized using the operators

$$
B_{l, p}^{\dagger}=\sum_{s=0}^{a-1} s_{p, s}^{l} b_{l, s}^{\dagger} .
$$

These are defined by the solutions of the eigenvalue equation

$$
\left[H, B_{l, p}^{\dagger}\right]=E_{l}^{p} B_{l, p}^{\dagger}
$$

The eigenvalues $E_{p}^{l}$ are the eigenvalues of the matrix $C^{l}$.

Hence, the Hamiltonian becomes

$$
H=\sum_{p=0}^{a-1} \sum_{l=1}^{m} E_{l}^{p} B_{l, p}^{\dagger} B_{l, p}-\frac{1}{2} h N
$$

Thus, the spectrum of this Hamiltonian is grouped into $a$ bands, each of which has $m=N / a$ levels. In the thermodynamic limit, keeping $a$ fixed and finite, the sums over the index $l$ become integrals.

\section{SOLUTIONS FOR $a=2$ AND 3}

\section{Case $a=2$ :}

For $a=2$, the eigenvalues of $C^{l}$ are

$$
\begin{aligned}
& E_{l}^{0}=h-\sqrt{v^{2}+\left(u^{2}-v^{2}\right) \cos [k(l)]^{2}}, \\
& E_{l}^{1}=h+\sqrt{v^{2}+\left(u^{2}-v^{2}\right) \cos [k(l)]^{2}} .
\end{aligned}
$$

The parameter $l$ takes the values $1 \leq l \leq N / 2$; hence, when $N \rightarrow \infty, 0 \leq k[l] \leq \pi$. Every value of the energy is doubly degenerate: $E_{l}^{i}=E_{N / 2-l}^{i}$. In Fig. 1 the energy of the two bands for the values $h=0, u=1$ and $v=0.5$ is shown. For $h=0$, the lower band has 


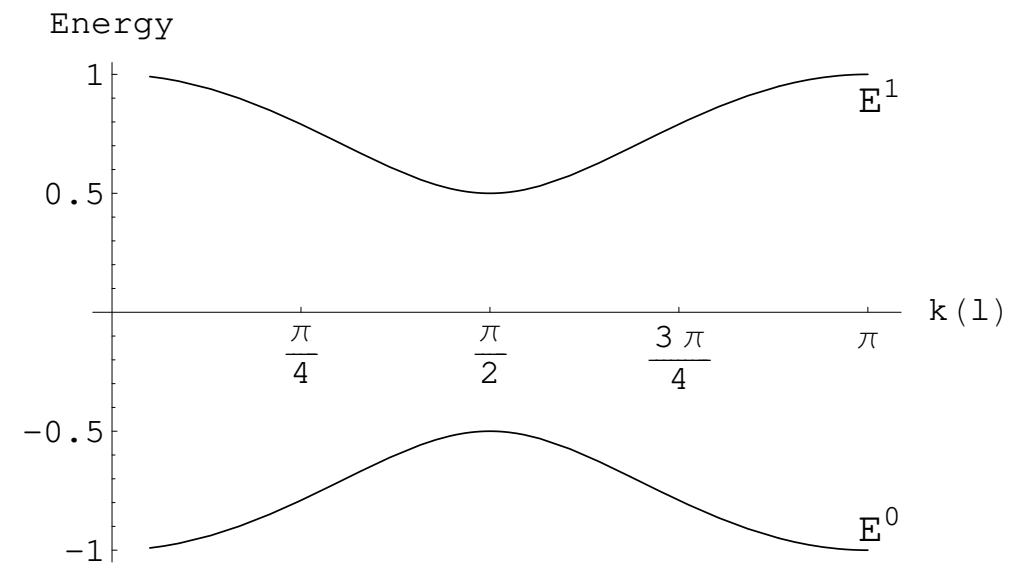

Figure 1: Energy of the two levels $E_{0}$ and $E_{1}$ for $u=1$ and $v=0.5$

$E^{0} \in[-u,-v]$, while the upper band has $E^{1} \in[v, u]$. The difference in energy between the two bands is $2 \min \{u, v\}$. When $u=v$ both energies are independent of $l$ and every band collapses into a single level with values $E_{0 / 1}=\mp u$.

Fig. 2 shows the difference,

$$
\Delta E=E_{l}^{1}-E_{l}^{0}=2 \sqrt{u^{2} \cos ^{2} k(l)+v^{2} \sin ^{2} k(l)}
$$

The the minimum of $\Delta E$ represents difference in energy between the lower and upper bands.

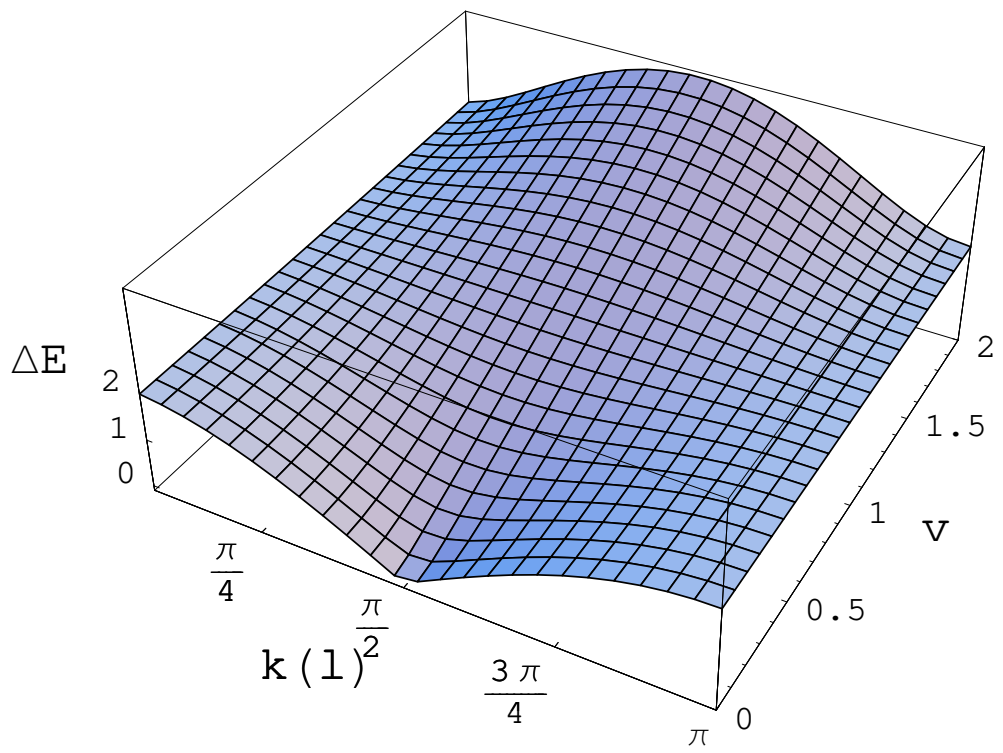

Figure 2: Energy difference between the two levels $E_{0}$ and $E_{1}$ 
Case $a=3$ :

In this case, the solutions for the eigenvalues of the $C^{l}$ are

$$
\begin{aligned}
& E_{l}^{0}=\frac{1}{48}\left(\frac{v^{(2)}}{f^{(2)}}-f^{(2)}\right) \\
& E_{l}^{1}=\frac{1}{96}\left((-1+i \sqrt{3}) \frac{v^{(2)}}{f^{(2)}}+(1+i \sqrt{3}) f^{(2)}\right), \\
& E_{l}^{2}=\frac{1}{96}\left((-1-i \sqrt{3}) \frac{v^{(2)}}{f^{(2)}}+(1-i \sqrt{3}) f^{(2)}\right) .
\end{aligned}
$$

where

$$
\begin{aligned}
f^{(1)} & =\left(27648-20736 v^{2}+6912 v^{3}\right) \cos [3 k(l)] \\
v^{(2)} & =-576-288 v^{2} \\
f^{(2)} & =\left(\frac{1}{2}\left(f^{(1)}+\sqrt{f^{(1)^{2}}+4 v^{(2)^{3}}}\right)\right)^{\frac{1}{3}}
\end{aligned}
$$

These coefficients are real.

In Fig. 3, the energy levels of the three bands $E^{0}, E^{1}$, and $E^{2}(27)$ are plotted as a function of $k(l)$ for four different values of $v$. The positions are marked on the $x$-axis where $k(l)$ is, for $l$ in the interval $[1, N / 3]$.

The width of the bands are shown in Fig. (4). They are given by the expressions

$$
W\left(E^{0}\right)=\frac{W\left(E^{1}\right)}{2}=W\left(E^{2}\right)=\left\{\begin{array}{ll}
\frac{-1-v+\sqrt{9-6 v+3 v^{2}}}{4} & \text { if } v>0 \\
\frac{1+v}{2} & \text { if } v<0
\end{array} .\right.
$$

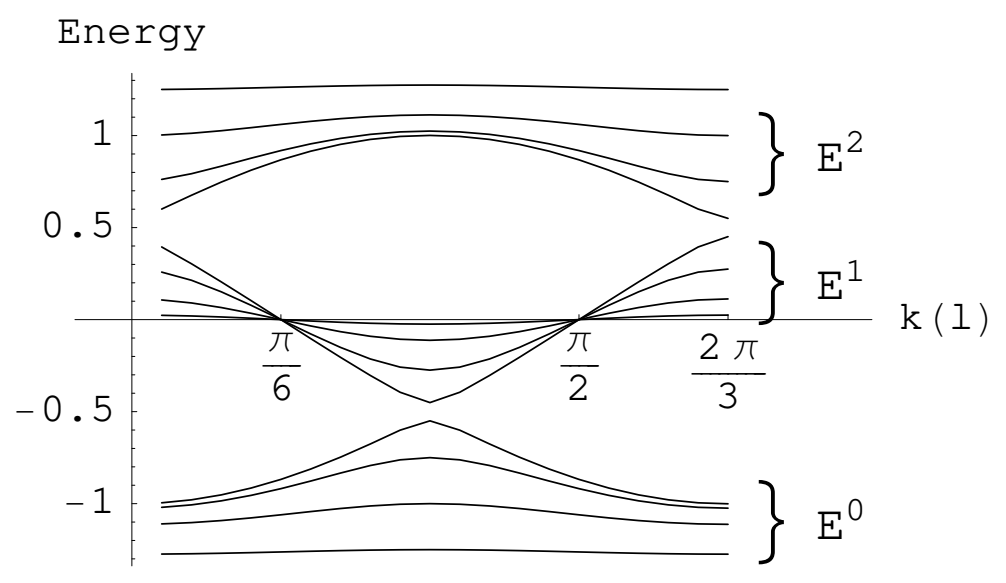

Figure 3: Energy of the three bands $E^{0}, E^{1}$ and $E^{2}$ for the values $u=1$, and $v=0.1,0.5,1.0$, and 1.5 plotted as a function of $k(l)$. 


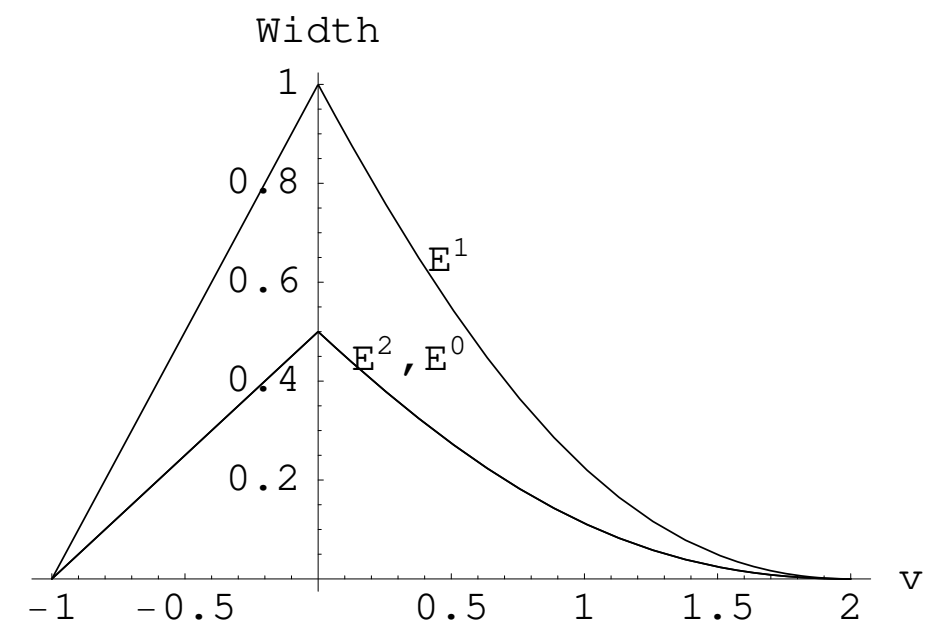

Figure 4: Energy band widths $W\left(E^{0}\right)=W\left(E^{2}\right)$ and $W\left(E^{1}\right)$

It can be seen from Fig.(4) that with increasing $v$, the width of each band increases when $v$ is negative and decreases when $v$ is positive. The width is zero for $v=-1$ and 2 , meaning that at these two values each band degenerates into a single level.

More generally, for an arbitrary value of $a$, the bands degenerate into single levels when $v$ and $u$ satisfy the condition

$$
\frac{v}{u}=-\frac{1}{\cos \left(\frac{2 \pi j}{a}\right)}, \quad j=1, \cdots, a .
$$

When this happens, the chain splits into a number of noninteracting parts. For example, $j=3$ yields the solution $v=-1$, and the chain is split into noninteracting subsystems with three sites each. If $j=1$ or 2 , then $v=2$ and produces a chain where only the sites in the positions $3 k$ and $3 k+1$ ( $k=$ integer $)$ are interacting.

The energy difference for three bands, $\Delta E=\min \left|E_{l}^{2}-E_{l^{\prime}}^{1}\right|=\min \left|E_{l^{\prime \prime}}^{1}-E_{l^{\prime \prime \prime}}^{0}\right|$, is given by

$$
\Delta E=\left|\frac{3+3 v-\sqrt{9-6 v+3 v^{2}}}{4}\right|
$$

$\Delta E$ over the interval $v=-1$ to 2 is shown in Fig. 5. At fixed $u=1$ and large $v, \Delta E$ is proportional to $v$ :

$$
\Delta E_{u=1, v \gg 2}=G v,
$$

where the constant of proportionality

$$
G=\frac{3-\sqrt{3}}{4} .
$$




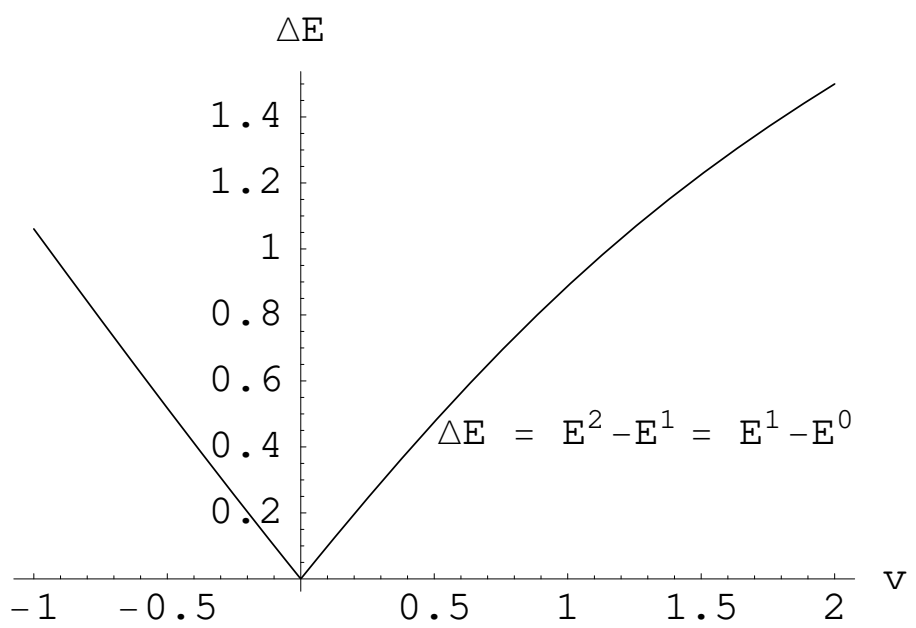

Figure 5: Energy difference for three bands $\Delta E=\min \left|E^{1}-E^{0}\right|=\min \left|E^{2}-E^{1}\right|$

\section{Case $a>3$ :}

Larger values of $a$ are straightforward to deal with. Numerical results for the energy bands with $a=12, u=10$ and $v=3$ are shown in Fig. 6.

As follows from (30), that the bands can degenerate into a single level only when $|v / u| \geq 1$. If we consider only $v= \pm u$, then both cases satisfy this condition when $a$ is even. However, if $a$ is odd, this is true only for $v=-u$. In all cases that satisfy the degeneracy condition, the chain is split into noninteracting subsystems containing $a$ sites.

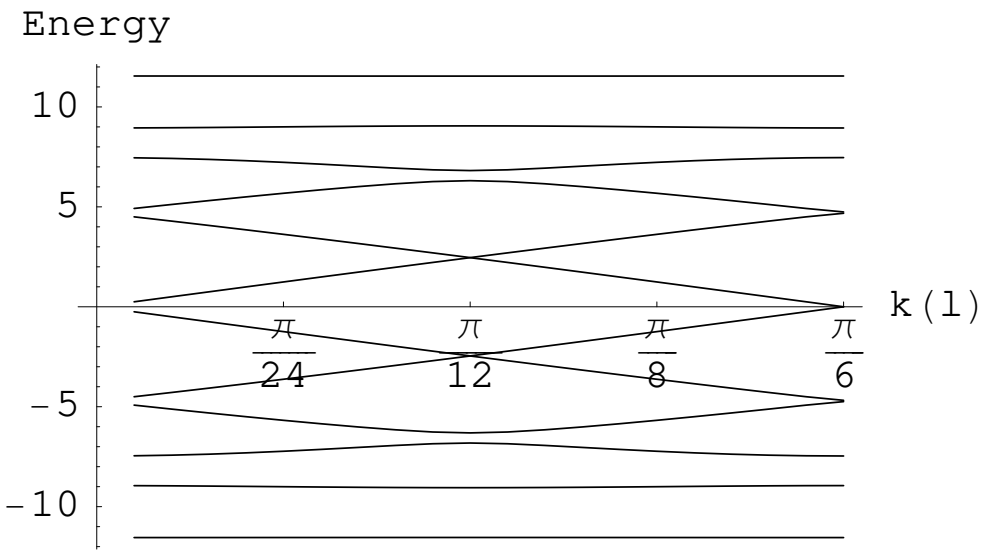

Figure 6: Energy Bands for $u=10, v=3$ and $a=12$ 


\section{THERMODYNAMIC FUNCTIONS}

The partition function is defined by

$$
Z=\operatorname{tr} e^{-\beta H}=\sum_{\mid \text {est }\rangle} e^{\beta h N / 2}\left\langle\operatorname{est}\left|\exp \left(-\sum_{p, l} E_{l}^{p} B_{l, p}^{\dagger} B_{l, p}\right)\right| \text { est }\right\rangle
$$

with $\beta=1 / T$, and $T$ being the temperature.

The most general state of the chain can be written as a tensorial product of the states of the sites which, in momentum space, are labelled by $\{l, p\}$. The state of every site, determined by the ket $|o\rangle_{l, p}$, can be occupied or empty:

$$
\mid \text { est }\rangle=\Pi_{l, p}|o\rangle_{l, p}, \quad \text { with } o=1, \text { (occupied) } o=0, \text { (empty) }
$$

Then, the trace is,

$$
\begin{aligned}
& \sum_{\text {lest }\rangle}\left\langle\operatorname{est}\left|\exp \left(-\beta \sum_{p, l} E_{l}^{p} B_{l, p}^{\dagger} B_{l, p}\right)\right| \text { est }\right\rangle \\
& \quad=\prod_{l, p}\left[\left\langle 0\left|\exp \left(-\beta E_{l}^{p} B_{l, p}^{\dagger} B_{l, p}\right)\right| 0\right\rangle+\left\langle 1\left|\exp \left(\beta E_{l}^{p} B_{l, p}^{\dagger} B_{l, p}\right)\right| 1\right\rangle\right]
\end{aligned}
$$

Due to the boundary conditions, the function $k(l)$ in $E_{l}^{p}$, in the product over $l, p$, is different when the number of occupied sites $N_{f}$ in $\mid$ est $\rangle$ is odd or even. However, when $N \rightarrow \infty$ the difference becomes negligible [16, 30], and

$$
Z=e^{\beta h N / 2} \prod_{l, p}\left[1+\exp \left(-\beta E_{l}^{p}\right)\right]
$$

This expression takes $\epsilon=0$ in $k(j)$ from (13) use it for $E_{l}^{p}$.

The thermodynamic free energy per site $f$ can be derived from the partition function as follows.

$$
f=-\frac{1}{\beta N} \ln Z=-\frac{h}{2}-\frac{1}{\beta N} \sum_{l, p} \ln \left[1+\exp \left(-\beta E_{l}^{p}\right)\right] .
$$

The thermodynamic limit is obtained by making $N \rightarrow \infty$ and keeping $a$ fixed and finite, whereupon the sums become integrals:

$$
\frac{1}{N} \sum_{l, p} \rightarrow \frac{1}{2 \pi} \sum_{p=0}^{a-1} \int_{0}^{2 \pi / a} d k
$$

When $a=2$ the free energy can be calculated analytically. To perform the integrals for this case, we first change the site parameter $l$ and define

$$
\begin{aligned}
& E_{l}=E_{l}^{0}=h-\lambda(l) \text { for }-\frac{N}{4}+1 \leq l \leq \frac{N}{4}, \\
& E_{l}=E_{l}^{1}=h+\lambda(l) \text { for }-\frac{N}{2}+1 \leq l \leq-\frac{N}{4} \text { and } \frac{N}{4}+1 \leq l \leq \frac{N}{2},
\end{aligned}
$$


where

$$
\lambda(l)=\sqrt{u^{2} \cos ^{2}(k(l))+v^{2} \sin ^{2}(k(l))}=\sqrt{v^{2}+\left(u^{2}-v^{2}\right) \cos ^{2}(k(l))} .
$$

Then, taking into account that $E_{l}$ is an even function of $l$, the free energy in the continuum limit is

$$
f=-\frac{h}{2}-\frac{1}{\pi \beta}\left(\int_{0}^{\pi / 2} \ln \left(1+e^{-\beta(h-\lambda(k))}\right) d k+\int_{\pi / 2}^{\pi} \ln \left(1+e^{-\beta(h+\lambda(k))}\right) d k\right) .
$$

Integrating by parts yields

$$
f=-\frac{h}{2}-\frac{1}{\beta}\left(\frac{1}{2} \ln \frac{1+e^{-\beta(h-v)}}{1+e^{-\beta(h+v)}}+\ln \left(1+e^{-\beta(h+u)}\right)\right)-\frac{1}{\pi}\left(I_{1}+I_{2}\right),
$$

where

$$
I_{1}=\int_{v}^{u} \arccos \left(-\sqrt{\frac{\lambda^{2}-v^{2}}{u^{2}-v^{2}}}\right) \frac{e^{-\beta\left(h^{+} \lambda\right)}}{1+e^{-\beta\left(h^{+} \lambda\right)}} d \lambda,
$$

and

$$
I_{2}=\int_{-u}^{-v} \arccos \left(\sqrt{\frac{\lambda^{2}-v^{2}}{u^{2}-v^{2}}}\right) \frac{e^{-\beta\left(h^{+} \lambda\right)}}{1+e^{-\beta\left(h^{+} \lambda\right)}} d \lambda .
$$

Both integrals can be performed by standard methods: see for example the appendix in Ref. [31]. Here, the function arccos is expanded as a series and integrated term by term. In the $I_{1}$ integral, we must considerer the cases $v>-h$ and $v<-h$. For $I_{2}$, the corresponding cases are $v>h$ and $v<h$. Then, $I_{1}$ in the first case is

$$
I_{1}=T\left(\frac{\pi}{2} \ln \left(1+e^{-\xi}\right)+\sum_{n=0} T^{\left(n+\frac{1}{2}\right)} a_{1,1}(n) i_{1,1}(n)\right),
$$

where

$$
\begin{aligned}
& a_{1,1}(0)=\frac{\sqrt{2 v}}{\sqrt{u^{2}-v^{2}}} \\
& a_{1,1}(1)=\frac{3 u^{2}+v^{2}}{6 \sqrt{2 v\left(u^{2}-v^{2}\right)^{3}}}, \\
& a_{1,1}(2)=\frac{-5 u^{4}+50 u^{2} v^{2}+3 v^{4}}{80 \sqrt{2 v^{3}\left(u^{2}-v^{2}\right)^{5}}} \\
& a_{1,1}(3)=\frac{7 u^{6}+7 u^{4} v^{2}+301 u^{2} v^{4}+5 v^{6}}{448 \sqrt{2 v^{5}\left(u^{2}-v^{2}\right)^{7}}}
\end{aligned}
$$

and

$$
i_{1,1}(n)=\int_{0}^{\frac{u-v}{T}=\infty} \frac{x^{\left(n+\frac{1}{2}\right)}}{1+e^{x+\xi}} d x=-\Gamma\left(\frac{3}{2}+n\right) \text { PolyLog }\left(\frac{3}{2}+n,-e^{-\xi}\right),
$$

with $\xi=(h+v) / T$. 
The expression for $I_{1}$ when $v<-h$ is more complicated. Following Refs. [32, 33], this can be written as follows.

$$
\begin{aligned}
I_{1}= & |h| \arccos \left(-\sqrt{\frac{h^{2}-v^{2}}{u^{2}-v^{2}}}\right)-\frac{\pi}{2} v-u \mathrm{E}\left[\arcsin \left(\frac{u}{h} \sqrt{\frac{h^{2}-v^{2}}{u^{2}-v^{2}}}\right), 1-\frac{v^{2}}{u^{2}}\right]+ \\
& \frac{\sqrt{\left(u^{2}-h^{2}\right)\left(h^{2}-v^{2}\right)}}{|h|}+\sum_{n=0} T^{n+1}\left[1-(-1)^{n}\right] a_{1,2}(n) i_{1,2}(n),
\end{aligned}
$$

where

$$
\begin{aligned}
& a_{1,2}(1)=-\frac{h}{\sqrt{\left(u^{2}-h^{2}\right)\left(h^{2}-v^{2}\right)}}, \\
& a_{1,2}(3)=-\frac{h\left[2 h^{6}-10 h^{2} u^{2} v^{2}+h^{4}\left(u^{2}+v^{2}\right)+3 u^{2} v^{2}\left(u^{2}+v^{2}\right)\right]}{6 \sqrt{\left(u^{2}-h^{2}\right)^{5}\left(h^{2}-v^{2}\right)^{5}}},
\end{aligned}
$$

and

$$
i_{1,2}(n)=\int_{0}^{\infty} \frac{x^{n}}{1+e^{x}} d x=\frac{2^{n}-1}{2^{n}} \Gamma(n+1) \zeta(n+1) .
$$

The first three elements of (51) are

$$
i_{1,2}(1)=\frac{\pi^{2}}{12}, \quad i_{1,2}(3)=\frac{7 \pi^{4}}{120}, \quad i_{1,2}(5)=\frac{31 \pi^{6}}{252}
$$

Once again, $|u-h| / T$ and $|h-v| / T$ are assumed to be large.

The $I_{2}$ integral when $h<v$ can also be performed using the tables provided in Refs. [32, 33], and a similar expansion to before. The result is

$$
\begin{aligned}
I_{2}= & (u-v)+u \mathrm{E}\left(\sqrt{1-\frac{v^{2}}{u^{2}}}\right)-T \frac{\pi}{2} \ln \left(1+e^{(h-v) / T}\right) \\
& -T \sum_{n=0} T^{n+\frac{1}{2}} a_{2,1}(n) i_{2,1}(n),
\end{aligned}
$$

where $a_{2,1}(n)=-a_{1,1}(n)$ from $(47)$, and $i_{2,1}(n)=i_{1,1}(n)$ from (48), with $\xi=(v-h) / T$.

The result for integral $I_{2}$ when $h>v$ is

$$
\begin{aligned}
I_{2}= & h \arccos \left(\sqrt{\frac{h^{2}-v^{2}}{u^{2}-v^{2}}}\right)+u \mathrm{E}\left[\arccos \left(\sqrt{\frac{h^{2}-v^{2}}{u^{2}-v^{2}}}\right), \sqrt{1-\frac{v^{2}}{u^{2}}}\right] \\
& +\sum_{n=0} T^{n+1}\left[1-(-1)^{n}\right] a_{2,2}(n) i_{2,2}(n),
\end{aligned}
$$

where $a_{2,2}(n)=-a_{1,2}(n)$ from $(50)$, and $i_{2,2}=i_{1,2}$ from $(51)$.

The free energy is obtained by substituting $I_{1}$ and $I_{2}$ into (43). Several examples are plotted in Fig. 7 for different values of $T$ and $v$, and for $u=1$, as functions of $h$. These 

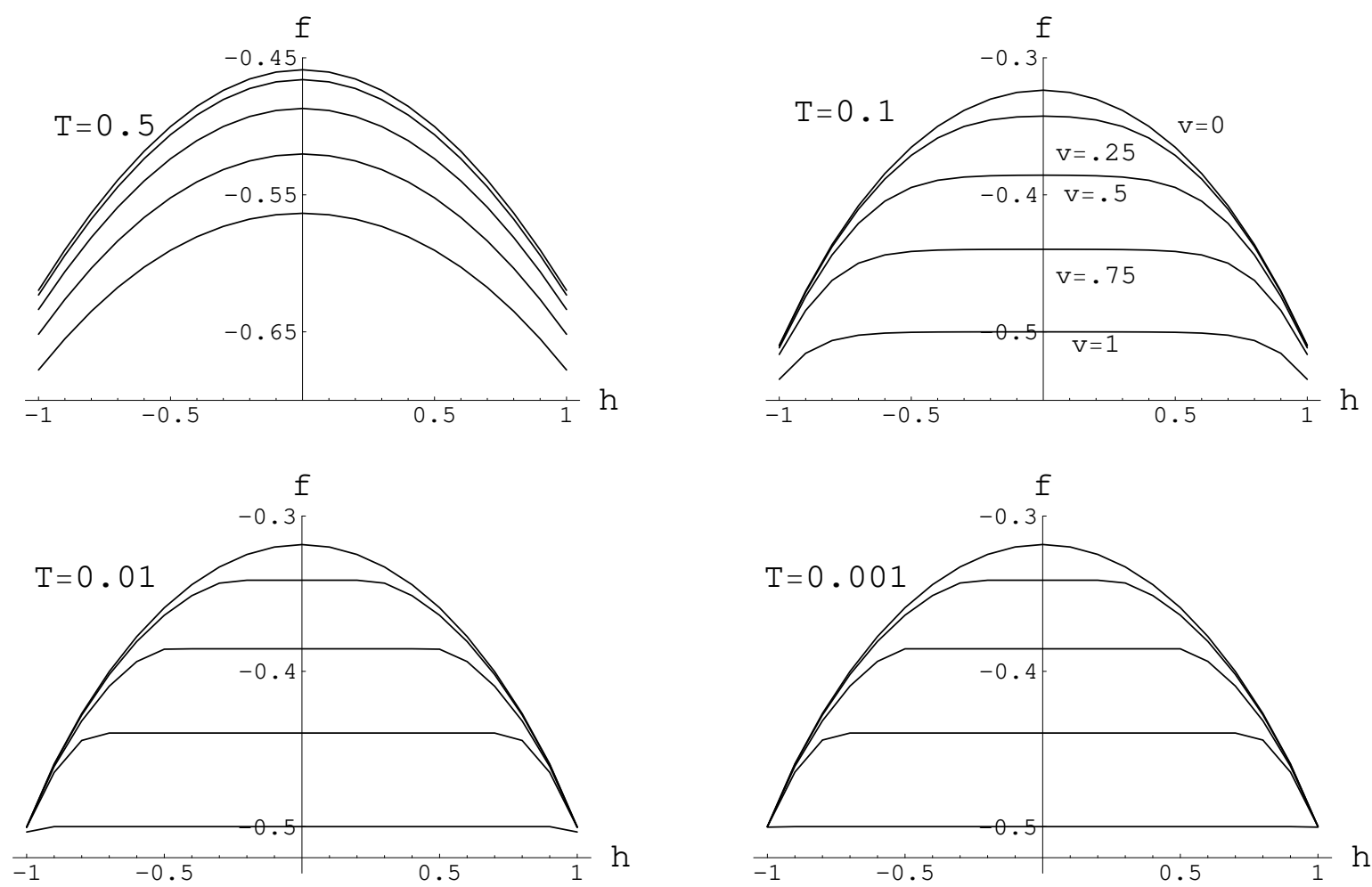

Figure 7: Free Energy plotted using $u=1$ and $v=0.0,0.25,0.5,0.75,1$.

show that, as implied by the Hamiltonian (3), the free energy is an even function of $h$. This property is also shown explicitly by an alternative expression to (42) for the free energy:

$$
f=-\frac{T}{\pi} \int_{0}^{\frac{\pi}{2}} \ln \left(2 \cosh \frac{h}{T}+2 \cosh \frac{\lambda(k)}{T}\right) d k .
$$

Notice that at low $T, f$ becomes nearly constant between $-v$ and $v$.

The entropy per site is defined by

$$
s=-\frac{\partial f}{\partial T}
$$

This can be obtained exactly from the expression for the free energy. To examine its behaviour we evaluate the partial derivative as follows.

$$
s=\frac{1}{\pi}\left[\int_{0}^{\frac{\pi}{2}} w\left(\frac{h-\lambda(x)}{T}\right) d x+\int_{\frac{\pi}{2}}^{\pi} w\left(\frac{h+\lambda(x)}{T}\right) d x\right],
$$

where the function $w(x)$ is

$$
w(x)=\ln \left(1+e^{-x}\right)+\frac{x e^{-x}}{1+e^{-x}} .
$$


This is an even function. Its magnitude is significant only when $x$ is small: $(w(5)=0.04$, $w(10)=0.0004)$. For large values of $|x|$, the expression simplifies to

$$
w(x)=|x| e^{-|x|}
$$

The integral can be evaluated in a similar manner to the one for the free energy. However, in this case the properties of the function $w$ allow us to examine the behaviour of $s$ in the low temperature limit. When $|h|<v$, the integrand in (56) can be approximated by (58). Taking the most significant term, yields the following solution.

$$
s(h, T)_{T \rightarrow 0} \approx \frac{1}{\pi} e^{-(v-|h|) / T} \rightarrow 0 .
$$

A similar approximation can be made for $|h|>u$ :

$$
s(h, T)_{T \rightarrow 0} \approx \frac{1}{\pi} e^{-(|h|-u) / T} \rightarrow 0 .
$$

To evaluate (56) for other values of $h$, we change the integration variable to

$$
y=\frac{h-\lambda(x)}{T}, \quad \text { and } \quad y=\frac{h+\lambda(x)}{T}
$$

respectively, in the two integrals. Hence, the entropy is

$$
\begin{aligned}
s= & \frac{1}{\pi}\left(\int_{(h-u) / T}^{(h-v) / T} w(y) \frac{T(h-T y)}{\sqrt{\left[u^{2}-(h-T y)^{2}\right]\left[(h-T y)^{2}-v^{2}\right]}} d y\right. \\
& \left.+\int_{(h+v) / T}^{(h+u) / T} w(y) \frac{T(T y-h)}{\sqrt{\left[u^{2}-(h-T y)^{2}\right]\left[(h-T y)^{2}-v^{2}\right]}} d y\right) .
\end{aligned}
$$

This integral has local maxima at $|h|=v$, where it can be approximated by

$$
s(|h|=v)=\frac{1}{\pi} \sqrt{\frac{T v}{2\left(u^{2}-v^{2}\right)}} \int_{0}^{\infty} \frac{w(y)}{\sqrt{y}} d y=0.6475 \sqrt{\frac{T v}{2\left(u^{2}-v^{2}\right)}} .
$$

A similar approximation can be made when $|h|=u$, where there is also a local maximum.

$$
s(|h|=u)=0.6475 \sqrt{\frac{T u}{2\left(u^{2}-v^{2}\right)}}
$$

When $v<|h|<u$-taking into account that $w(x)$ has significant values around $x \approx 0$, and is rapidly decreasing otherwise $-s$ can be approximated by

$$
s=\frac{1}{\pi} \frac{T|h|}{\sqrt{\left(u^{2}-h^{2}\right)\left(\left(h^{2}-v^{2}\right)\right.}} \int_{-\infty}^{\infty} w(y) d y=\frac{\pi T|h|}{3 \sqrt{\left(u^{2}-h^{2}\right)\left(h^{2}-v^{2}\right)}} .
$$


Here, we can use

$$
\frac{1}{\pi} \int_{0}^{\infty} \frac{w(x)}{\sqrt{x}} d x=0.6475, \quad \frac{1}{\pi} \int_{-\infty}^{\infty} w(x) d x=\frac{\pi}{3} .
$$

Thus, combining all of the above, the entropy is

$$
s(h, T)_{T \rightarrow 0}=\left\{\begin{array}{ll}
\frac{1}{\pi} e^{-(v-|h|) / T} \quad 0 & \text { if }|h|<v \\
0.6475 \sqrt{\frac{T v}{2\left(u^{2}-v^{2}\right)}} & \text { if }|h|=v \\
\frac{\pi T|h|}{3 \sqrt{\left(u^{2}-h^{2}\right)\left(h^{2}-v^{2}\right)}} & \text { if } u>|h|>v . \\
0.6475 \sqrt{\frac{T u}{2\left(u^{2}-v^{2}\right)}} & \text { if }|h|=u \\
\frac{1}{\pi} e^{-(|h|-u) / T} \rightarrow 0 & \text { if }|h|>u
\end{array} .\right.
$$

The entropy is plotted using four different values of $v$, each at three different temperatures in Fig. 8. Fig. 9 shows logarithmic graphs of the temperature dependence of the entropy when $u=1$ and $v=0.5$, for five values of values $h$.

The specific heat per site is

$$
C(h, T)_{T \rightarrow 0}=T \frac{\partial s}{\partial T}=\left\{\begin{array}{ll}
\left(\frac{v-|h|}{T}\right) e^{-(v-|h|) / T} & \text { if }|h|<v \\
0.3238 \sqrt{\frac{T v}{2\left(u^{2}-v^{2}\right)}} & \text { if }|h|=v \\
\frac{\pi T|h|}{3 \sqrt{\left(u^{2}-h^{2}\right)\left(h^{2}-v^{2}\right)}} & \text { if }|h|>v \\
0.3238 \sqrt{\frac{T u}{2\left(u^{2}-v^{2}\right)}} & \text { if }|h|=v \\
\frac{|h|-u}{T} e^{-(|h|-u) / T} & \text { if }|h|>u
\end{array} .\right.
$$

It can be seen that both functions (the entropy and the specific heat) decay exponentally as $e^{-1 / T}$ to zero when $T \rightarrow 0$, for $|h|<v$ and $|h|>u$. This also shows that the internal parameters of the system, $v$ and $u$, can be derived by measuring the specific heat as a function of $h$.

Conclusions for other values of periodicity $a$ can be generalised from this result. The specific heat will be exponentially small for values of $h$ between the several energy bands. Thus, measurements will provide information about the periodicity and its amplitude. 


\section{Acknowledgments}

We would like to thank Dr. A. Cruz for his comments. This work has been supported by the CICYT grants BFM2000-1057 and FPA2000-1252.

[1] J. W. Bray, H. R. Hart Jr., L. V. Interrante, I. S. Jacobs, J. S. Kasper, G. D. Watkius, S. H. Wee, and J. C Bonner. Phys. Rev. Lett., $35: 744$, (1975).

[2] M. Hase, I. Teraski, and K. Uchinokura. Phys. Rev. Lett., 70 :3651, (1993).

[3] M. Hase, I. Teraski, K. Uchinokura, K. Tokunaga, N. Miura N., and H. Obara. Phys. Rev. B, $48: 9161,(1993)$.
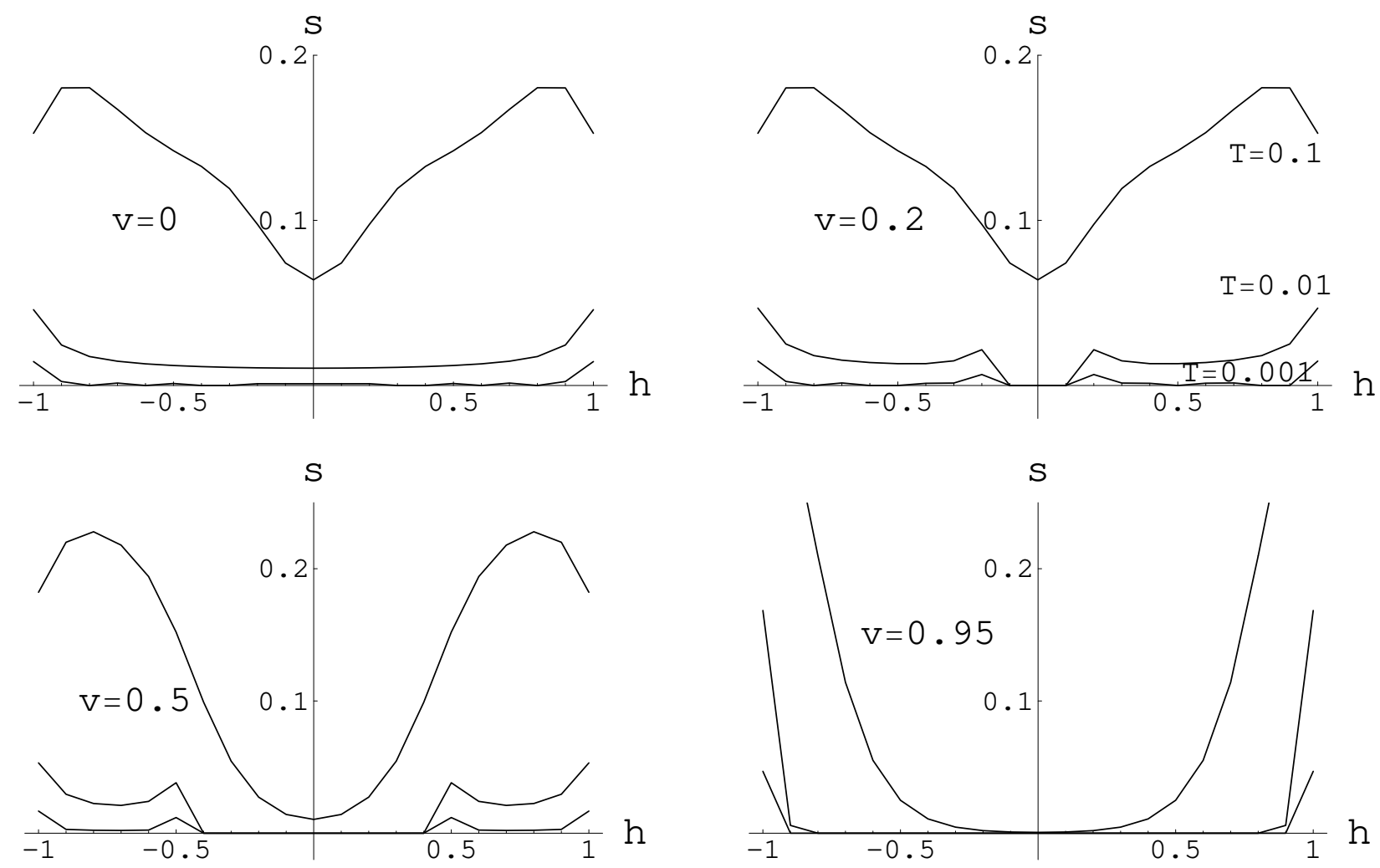

Figure 8: Entropy plotted using $u=1$ and $T=0.1,0.01,0.001$. The ordering of the lines for $v=0.2$ is the same in the other three cases. 

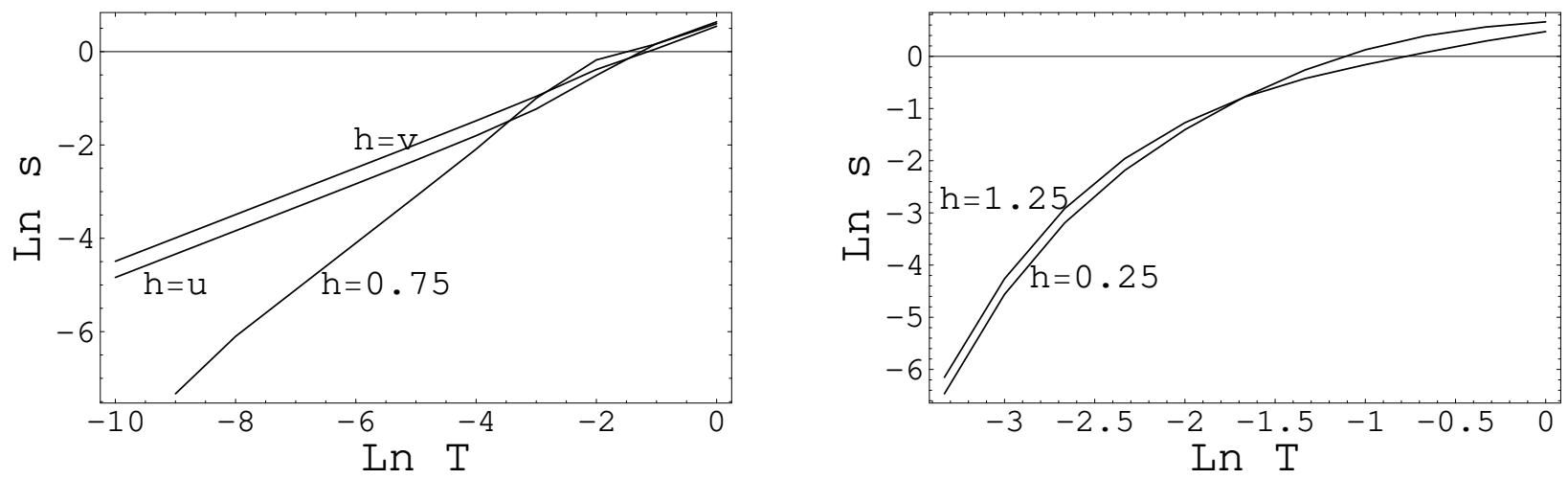

Figure 9: Graphs of $\ln S$ versus $\ln T$ plotted using $u=1$ and $v=0.5$, for five values of $h$. Left: $h=1,0.75,0.5 ;$ and right: $h=1.25,0.25$.

[4] J. P. Boucher and L. P. Regnault. Physique, I6 :1939, (1996).

[5] D. C. Johnston, J. W. Johnson, D. P. Gorshornand, and A. J. Jacobson. Phys. Rev. B, 35 :219, (1987).

[6] H. P. Nightingale and H. W. Blöte. Phys. Rev. B, 33 :659, (1986).

[7] S. R. White and D. A. Huse. Phys. Rev B, 33 :3844, (1993).

[8] D. Schechtman, I. Blech, D. Gratias, and J. W. Cahn. Phys. Rev. Lett., 53 :1951, (1984).

[9] D. Levine and P. J. Steinhardt. Phys. Rev. Lett., 53 :2477, (1984).

[10] J. M. Luck. Jour. of Stat. Phys., 72 :417, (1993).

[11] E. Daggoto and T. M. Rice. Science, 271 :618, (1996).

[12] T. N. Nguyen, P. A. Lee, and H. C. Loye. Science, 271 :489, (1996).

[13] P. Gambardella, A. Dallmeyer, K. Maiti, M. C. Malagoli, W. Eberhardt, K. Kern, and C. Carbone. Nature, 416 :301, (2002).

[14] I. G. Bostrem, A. S. Boyarchenkov, A. A. Konovalov, A. S. Ovchinnikov, and V. E. Sinitsyn. J. Exp. and Theoret. Phys., $97: 615,(2003)$.

[15] K. Okamoto. Prog. Theoret. Phys. Supp., 145 :113, (2002).

[16] E. Lieb, T. Schultz, and D. Mattis. Ann. of Phys., 16:407, (1961).

[17] P. Jordan and E. Wigner. Z. Physik, 47 :631, (1928).

[18] J. H. H. Perk, H. W. Capel, M. J. Zuilhof, and Th. J. Siskens. Physica A, 81 :319, (1975).

[19] J. H. Taylor and G. Müller. Physica A, 130 :1, (1985).

[20] D. S. Fisher. Phys. Rev. Lett., 69 :534, (1992). 
[21] J. M. Luck and J. M. Nieuwenhuizen. Europhys. Lett, 2 :257, (1986).

[22] A. P. Young and H. Rieger. Phys. Rev. B, 53 :8486, (1984).

[23] O. Derzhko, J. Richter, T. Krokhmalskii, and O. Zaburannyi. Phys. Rev E, 69 :066112, (2004).

[24] O. Derzhko and J. Richter. Phys. Rev B, 59 :100, (1999).

[25] O. Derzhko, J. Richter, and O. Zaburannyi. Physica A, 282 :495, (2000).

[26] L. L. Gonçalves and J. P. de Lima. J. Magn. Magn. Mater, 140 :1606, (1995).

[27] J. P. de Lima and L. L. Gonçalves. Physica A, 311 :458, (2002).

[28] J. P. de Lima and L. L. Gonçalves. J. Magn. Magn. Mater, 206 :135, (1999).

[29] J. P. de Lima, T. F. Alves, and L. L. Gonçalves. cond-mat/0501525.

[30] M. Takahashi. Themodynamic of One-Dimensional Solvable Model. Cambridge University Press, Cambridge, U.K., (1999).

[31] R. K. Pathia. Statistical Mechanics. Butterworth and Heinemann, Oxford, U.K., (1996).

[32] P. F. Byrd and M. D. Friedman. Handbook of Elliptic Integrals for Engineers and Scientists. Springer-Verlag, New York, Heidelberg, Berlin, (1971).

[33] A. P. Prudnikov, Y. A. Brychkov, and O. I. Marichev. Integrals and Series. Gordon and Breach Science Publishers, New York, (1986). 